\title{
ON RELATIVE NORMAL COMPLEMENTS IN FINITE GROUPS. II
}

\author{
HENRY S. LEONARD, JR.
}

\begin{abstract}
Given a finite group $G$ and subgroups $H$ and $H_{0}$ with $H_{0} \triangleleft H$, we let $\pi$ denote the set of prime divisors of $\left(H: H_{0}\right)$, and we denote this configuration by $\left(G, H, H_{0}, \pi\right)$. Pamela Ferguson has shown that if $H / H_{0}$ is solvable, then under certain conditions there exists a unique relative normal complement $G_{0}$ of $H$ over $H_{0}$. In this paper we give alternative proofs of her two theorems.
\end{abstract}

Let $G$ be a finite group and $\pi$ a set of primes. The complementary set of primes will be denoted by $\pi^{\prime}$. Let $\pi(G)$ denote the set of prime divisors of $|G|$. We call $G$ a $\pi$-group if $\pi(G) \subseteq \pi$. An element $x$ of $G$ is a $\pi$-element if $\langle x\rangle$ is a $\pi$-group. Every element $x$ of $G$ has a unique decomposition, $x=x_{\pi} x_{\pi^{\prime}}=x_{\pi^{\prime}} x_{\pi}$, into a $\pi$-element $x_{\pi}$ and a $\pi^{\prime}$-element $x_{\pi^{\prime}}$. Both $x_{\pi}$ and $x_{\pi^{\prime}}$ are powers of $x$. Two elements $x$ and $y$ belong to the same $\pi$-section of $G$ if their $\pi$-parts $x_{\pi}$ and $y_{\pi}$ are conjugate in $G$. If $S$ is a subset of $G$ we let $S^{G, \pi}$ denote the union of all $\pi$-sections of $G$ that intersect $S$.

We let $\left(G, H, H_{0}, \pi\right)$ denote the following configuration. Let $G$ be a finite group with subgroups $H$ and $H_{0}$ such that $H_{0} \triangle H$ and $\pi=\pi\left(H / H_{0}\right)$. Given this, and given a subgroup $G_{0}$ of $G, G_{0}$ is called a relative normal complement of $H$ over $H_{0}$ if $G_{0} \triangle G, G=G_{0} H$, and $H_{0}=G_{0} \cap H$.

For $\left(G, H, H_{0}, \pi\right)$ we consider the following conditions:

(A) Any two $\pi$-elements of $H-H_{0}$ that are $G$-conjugate are $H$-conjugate.

$\left(\mathrm{B}_{0}\right)$ For each $\pi$-element of $H-H_{0}$ we have $C_{G}(x)=O_{\pi^{\prime}}\left(C_{G}(x)\right) C_{H}(x)$.

(C) $\left|\left(H-H_{0}\right)^{G, \pi}\right|=(G: H)\left|H-H_{0}\right|$.

Pamela Ferguson has proved the following two theorems [1].

THEOREM 1. If $\left(G, H, H_{0}, \pi\right)$ satisfies conditions $\left(\mathrm{B}_{0}\right)$ and $(\mathrm{C})$ and $H / H_{0}$ is solvable, then there exists a unique relative normal complement $G_{0}$ of $H$ over $H_{0}$ and $G_{0}=G-\left(H-H_{0}\right)^{G, \pi}$.

THEOREM 2. If $\left(G, H, H_{0}, \pi\right)$ satisfies conditions (A) and $\left(\mathrm{B}_{0}\right)$ and $H / H_{0}$ is solvable, then there exists a unique relative normal complement $G_{0}$ of $H$ over $H_{0}$ and $G_{0}=G-\left(H-H_{0}\right)^{G, \pi}$.

Theorem 2 was proved by Reynolds [3, Theorem 2] in the case that $H / H_{0}$ is a p-group.

It is the purpose of this paper to give alternative proofs of these theorems. First we prove a lemma to the effect that the two theorems are equivalent.

Received by the editors July 26, 1982.

1980 Mathematics Subject Classification. Primary 20D20.

Key words and phrases. Finite groups, normal complement, $\pi$-subgroups. 
LEMmA. If $\left(G, H, H_{0}, \pi\right)$ satisfies condition $\left(\mathrm{B}_{0}\right)$, then condition (A) is satisfied if and only if condition $(\mathrm{C})$ is satisfied.

Proof. Suppose $x \in H-H_{0}$ and $y \in H$, and suppose $x_{\pi}$ and $y_{\pi}$ are $H$-conjugate. Since $H / H_{0}$ is a $\pi$-group, $x_{\pi^{\prime}}$ and $y_{\pi^{\prime}} \in H_{0}$ and $x_{\pi} \in H-H_{0}$. Hence, $y_{\pi} \in H-H_{0}$, so $y \in H-H_{0}$. Thus $H-H_{0}$ is a union of $\pi$-sections of $H$.

Condition (A) holds if and only if, for every $\pi$-section $S$ of $G$, either $S \cap(H-$ $\left.H_{0}\right)=\varnothing$ or $S \cap\left(H-H_{0}\right)$ is a $\pi$-section of $H$. Hence, [2, Lemma 3.4] states that condition (A) holds if and only if condition (C) holds (assuming condition $\left(\mathrm{B}_{0}\right)$ ), and our proof is complete.

Now we prove Theorem 2 (and hence Theorem 1).

Proof of Theorem 2. Let $\left(G, H, H_{0}, \pi\right)$ be given satisfying the hypotheses of Theorem 2. We may assume that the theorem holds for groups of order less than $|G|$. Since $H / H_{0}$ is solvable, there is a prime $p \in \pi$ such that $H$ has a normal subgroup $H_{1}$ of index $p$ with $H_{1} \supseteq H_{0}$.

It is easily verified that $H-H_{1}$ is a union of $p$-sections of $H$. If $x$ and $y$ are $G$-conjugate $p$-elements of $H-H_{1}$, then, by hypothesis, they are $H$-conjugate. Thus $\left(G, H, H_{1},\{p\}\right)$ satisfies condition (A). If $x$ is a $p$-element of $H-H_{1}$, then, by hypothesis,

$$
C_{G}(x)=O_{\pi^{\prime}}\left(C_{G}(x)\right) C_{H}(x)
$$

But $p \in \pi$, so

$$
C_{G}(x)=O_{p^{\prime}}\left(C_{G}(x)\right) C_{H}(x)
$$

Thus $\left(G, H, H_{1},\{p\}\right)$ satisfies condition $\left(\mathrm{B}_{0}\right)$. Therefore Reynolds' theorem [3, Theorem 2] implies that in $G$ there is a normal complement $G_{1}$ of $H$ over $H_{1}$.

Let $\pi_{1}=\pi\left(H_{1} / H_{0}\right)$. We now verify that $\left(G_{1}, H_{1}, H_{0}, \pi_{1}\right)$ satisfies the hypotheses of Theorem 2. Let $x$ and $y$ be $G_{1}$-conjugate $\pi_{1}$-elements of $H_{1}-H_{0}$. By hypothesis they must be $H$-conjugate. Hence, there exist $g_{1} \in G_{1}$ and $h \in H$ such that $y=x^{g_{1}}$ $=x^{h}$. Hence, $g_{1} h^{-1} \in C_{G}(x)$, and hypothesis $\left(\mathrm{B}_{0}\right)$ implies there exist $g_{2} \in O_{\pi^{\prime}}\left(C_{G}(x)\right)$ and $k \in C_{H}(x)$ such that $g_{1} h^{-1}=g_{2} k$. But $O_{\pi^{\prime}}\left(C_{G}(x)\right) \subseteq G_{1}$ since $G_{1}$ is a normal subgroup of $G$ of index $p$. Therefore $g_{2}^{-1} g_{1}=k h \in G_{1} \cap H=H_{1}$, so $g_{2}^{-1} g_{1}=h_{1} \in H_{1}$ for some $h_{1}$. Since $g_{2} \in C_{G}(x)$, we have $y=x^{g_{1}}=x^{g_{2}^{-1} g_{1}}=x^{h_{1}}$. Thus $x$ and $y$ are $H_{1}$-conjugate, so $\left(G_{1}, H_{1}, H_{0}, \pi\right)$ satisfies condition (A).

Let $x$ be a $\pi_{1}$-element of $H_{1}-H_{0}$. Since $\pi_{1} \subseteq \pi$ and $G_{1}$ is a normal subgroup of $G$ of index $p, O_{\pi^{\prime}}\left(C_{G}(x)\right)$ is a normal $\pi_{1}^{\prime}$-subgroup of $C_{G_{1}}(x)$. Therefore hypothesis $\left(\mathrm{B}_{0}\right)$ implies

$$
\begin{aligned}
C_{G_{1}}(x) & =O_{\pi^{\prime}}\left(C_{G}(x)\right) C_{H}(x) \cap G_{1} \\
& =O_{\pi^{\prime}}\left(C_{G}(x)\right)\left(C_{H}(x) \cap G_{1}\right)=O_{\pi^{\prime}}\left(C_{G}(x)\right) C_{H_{1}}(x),
\end{aligned}
$$

so that $\left(G_{1}, H_{1} H_{0}, \pi_{1}\right)$ satisfies condition $\left(\mathrm{B}_{0}\right)$. Hence, by our induction hypothesis, there is a relative normal complement $G_{0}$ in $G_{1}$ of $H_{1}$ over $H_{0}$, and $G_{0}=G_{1}-$ $\left(H_{1}-H_{0}\right)^{G_{1}, \pi_{1}}$. 
Since $G=G_{1} H, G_{1} \triangle G$, and $H_{1}-H_{0}$ is a normal subset of $H$, it is easily seen that $\left(H_{1}-H_{0}\right)^{G_{1}, \pi_{1}}$ is a normal subset of $G$ and, hence, that $G_{0} \Delta G$. We have

$$
G=G_{1} H, \quad G_{1} \cap H=H_{1}, \quad \text { and } \quad G_{1}=G_{0} H_{1}, \quad G_{0} \cap H_{1}=H_{0} .
$$

Therefore

$$
G=G_{1} H=G_{0} H_{1} H=G_{0} H
$$

and

$$
G_{0} \cap H=G_{0} \cap G_{1} \cap H=G_{0} \cap H_{1}=H_{0},
$$

so $G_{0}$ is a relative normal complement in $G$ of $H$ over $H_{0}$.

It remains to show that $G_{0}=G-\left(H-H_{0}\right)^{G, \pi}$. According to the above lemma,

$$
\left|\left(H-H_{0}\right)^{G, \pi}\right|=(G: H)\left|H-H_{0}\right|,
$$

so

$$
\left|G-\left(H-H_{0}\right)^{G, \pi}\right|=(G: H)\left|H_{0}\right| \text {. }
$$

Therefore [2, Proposition 2.2] yields our equation for $G_{0}$, and the proof of the theorem is complete.

\section{REFERENCES}

1. P. Ferguson, Relative normal complements in finite groups, Proc. Amer. Math. Soc. 87 (1983), 38-40.

2. H. Leonard, On relative normal complements in finite groups, Arch. Math. (Basel) (to appear).

3. W. F. Reynolds, Isometries and principal blocks of group characters, Math. Z. 107 (1968), 264-27().

Department of Mathematical SCiEnCES, NoRThern Illinois University, DeKalb, Illinois 60115 